\title{
TUBERCULOSIS NFANTIL
}

\author{
Por e] Dr, JULIO ESPINOZA S. \\ Instituto de Anatom.a-Patológica del Hospital de Niñios "M. Atriarín".
}

(Continuasión)

IV

Perforación de órganos torácicos por chancros, cavernas y ganglios.

En nuestro material de 478 necropsias con tuberculosis, bemos encontrado 50 perforaciones de chancros, cavernas primarias y no primarias y de ganglios caseificados, a diversos órgatios torácicos, lo cual significa una proporción global de $10.46 \%$. Este porcentaje podría parecernos elevado si consideramos la opinión de autores extranjeros como Pehu y Dufourt ${ }^{14}$ y Huebschmann ${ }^{15}$, que consideran esta complicación como excepcional. Koleman ${ }^{16}$, de Londres, la ha observado en su material de necropsias en el $1 \%$. Sin embargo, Ghon ${ }^{17}$ da una cifra de $17.7 \%$ y Görgenyi y Kassay ${ }^{24}$, del $47.1 \%$, también en material de autopsia.

La presencia de las perforaciones según la edad de los casos en que éstas se han proóucido (cuadro $\mathrm{N}^{\circ} 13$ ), es evidentemente mayor en los 4 primeros años de la vida, época en que tiene lugar la infección primaria tuberculosa y las adenopatias caseosas subsecuentes, las cuales, según en seguida veremos, son las que suministran la mayor fuente de origen de estas complicaciones. Vale la pena señalar que en el tercer año, el porcentaje alcanza al 17.74, cifra que, como recién anotamos, es dada por Ghon para este accidente.

Según el cuadro $N^{\circ} 14$, de las 50 perforaciones, sólo 7 (14\%) son producidas por elementos ajenos al complejo primario, como son cavernas de reinfección, crónicas o agudas y como causa printipal de este accidente aparecen los ganglios caseificados tráqueo-bronquiales $(70 \%)$. Entre estos últimos, los intertráqueos-brónquicos han participado 24 veces entre 35 perforaciones, o sea, el $68.57 \%$ de los casos de perforación por

Cuadro $\mathrm{N}^{2} 13$

Porcentaje de perforaciones según la edad.

\begin{tabular}{lccc} 
Edades & $\begin{array}{c}\mathrm{N}^{\circ} \mathrm{de} \\
\text { necrop. } \\
\text { con tbc. }\end{array}$ & $\begin{array}{c}\mathrm{N}^{\mathrm{d}} \mathrm{de} \\
\text { perfora- } \\
\text { ciones }\end{array}$ & $\begin{array}{c}\text { Porcen- } \\
\text { taje }\end{array}$ \\
\hline
\end{tabular}

\begin{tabular}{|c|c|c|c|}
\hline 0 a $12 \mathrm{~m}$. & 94 & 11 & 11.70 \\
\hline 12 a $24 \mathrm{~m}$. & 99 & 16 & 16.66 \\
\hline 2 a 3 a. & 62 & 11 & 17.74 \\
\hline 3 a 4 a. & 37 & 5 & 1351 \\
\hline 4 a 6 a. & 52 & 1 & 1.92 \\
\hline 6 a 8 a. & 45 & 2 & 4.44 \\
\hline 8 a $10 \mathrm{a}$. & 34 & 1 & 2.94 \\
\hline 10 a 12 a. & 47 & 2 & 4.25 \\
\hline 12 a 14 a. & 6 & 0 & 0.00 \\
\hline 14 a 16 a. & 2 & 1 & 50.00 \\
\hline & 478 & 50 & \\
\hline
\end{tabular}

Cuadro $\mathrm{N}^{\circ} 14$

Perforaciones de órganos torácicos por chancros $y$ zanglios.

\begin{tabular}{lcr}
\hline & $\begin{array}{c}N^{0} \text { de } \\
\text { casos }\end{array}$ & $\begin{array}{c}\text { Porcen } \\
\text { taje }\end{array}$ \\
\hline Chancros primarios & 2 & 4 \\
Cavernas primarias & 6 & 12 \\
Cavernas no prkmarias & 7 & 14 \\
Ganglios caselficadogs & 35 & 70 \\
\multicolumn{1}{c}{ Total } & 50 &
\end{tabular}


ganglios caseificados (cuadro $N^{\circ} 15$ ). Creemos que esta mayor participación de los ganglios inter-tráqueo-brónquicos en el proceso que comentamos, es debida-a que, según Rouviere ${ }^{18}$ estos ganglios son tributarios absolutos de las zonas inferiores de ambos pulmones y parciales de las medianas y están en estrecha relación con los ganglios paratraqueales derechos, donde desaguan, los cuales drenan el resto del pulmón derecho, en tal forma que los ganglios inter-tráqueo-brónquicos son afectados con mucha mayor frecuencia que el resto de los ganglios tráqueo-bronquiales en cualquiera infección pulmonar.

Cuadro $N^{9} 15$

\section{Grupes ganglionares causantes de perforactones.}

\begin{tabular}{lrrrr}
\hline & & & $\begin{array}{c}\text { Porcen- } \\
\text { taje }\end{array}$ \\
\hline Intertráqueabrónquicos & 24 & veces & $\mathbf{6 8 . 5 7}$ \\
Hiliares (pulmón) & 6 & $"$ & $\mathbf{1 7 . 1 4}$ \\
Paratraqueales inf. D. & 4 & & $\mathbf{1 1 . 4 2}$ \\
Paratraqueales inf. I. & 1 & & $\mathbf{2 . 8 0}$ \\
\multicolumn{1}{c}{ Total } & & &
\end{tabular}

Además, debemos recordar que el chancro primario tuberculoso se encuentra en el $60 \%$ de los casos en el pulmón derecho. A este último factor también debemos atribuir el que en 7 casos de perforación bronquial por chancro o caverna primaria, 6 lo sean del bronquio derecho y sólo uno del izquierdo, experiencia ya obtenida por Schwarz, Peña y Meneghello ${ }^{19}$, en 1944.

Es de interés, además, comentar la adenopatía tuberculosa de los casos con perforación, desde el punto de vista de la extensión de la enfermedad ganglionar. En los 34 casos de adenopatía taberculosa, que dieron lugar a 35 perforaciones, hubo 1 caso que produjo 2 perforaciones (Necropsia $N^{9} 613 / 45$ ): fueron muy raros aquéllos, on que los ganglios correspondientes al complejo primario eran los únicos afectados por la ruberculosis caseosa (cuadro $\mathrm{N}^{\circ} 16$ ).
Treinta y un casos $(91 \%)$ de los 34 a que nos estamos refiriendo, presentaban una adenopatía tuberculosa caseosa extendida desde la región tráqueobronquial, asiento del chancro, bacia las regiones cervical y abdominal, a sea, existía en un alto porcentaje una tubercalosis caseosa generalizada de los ganglios. Esto nos da a entender la gravedad de este tipo de adenopatía en el terreno de las perforaciones de los órganos toráci$\cos$ con sus consecuencias inmediatas Cuadro N: 16

Extensión de la adenopatía tuberenlosa en los casos de perforación por ganglios caseificados.

\begin{tabular}{lcc}
\hline & $\begin{array}{c}\mathrm{N}^{\circ} \mathrm{de} \\
\text { casos }\end{array}$ & $\%$ \\
\hline $\begin{array}{l}\text { Del complejo primario } \\
\text { Torácicos y cervicales }\end{array}$ & 3 & $\mathbf{8 . 8 2}$ \\
$\begin{array}{l}\text { Torácicos, cervicales y abdo- } \\
\text { minales }\end{array}$ & 1 & 2.94 \\
Torácicos y abdominales & 16 & 47.05 \\
\multicolumn{1}{c}{ Total } & -34.17
\end{tabular}

(diseminación broncógena), además de otras a que dan lugar y a las cuales nos referiremos más adelante (diseminaciones hematógenas).

Respecto de los órganos afectados por las perforaciones (cuadro $\mathrm{N}^{\circ} 17$ ), encontramos que los bronquios son los más comprometidos, figurando en primer $\mathrm{lu}^{-}$ gar el derecho con el $56 \%$ y el izquierdo con el $28 \%$ del total de las perforaciones. La causa del predominio de los bronquios derechos en el accidente que comentamos, la atribuimos, como decíamos más arriba, a la localización preferente del complejo primario en el pulmón derecho $(60 \%)$ y al mayor tamaño y número de los ganglios inter-tráqueo-brónquicos de este lado.

De los 43 casos en que hubo perforación bronquial y de la tráquea, se produjo diseminación canalicular en 33 , o sea, en el $76.74 \%$. De estos 33 casos, 29 presentaban también tubereulosis ulcerativa del intestino $(87.87 \%)$, pero 
Cuadro $\mathrm{N}^{\circ} 17$

Organos afectados por las perforaciones y porcentajes respectivos.

\begin{tabular}{ccccc} 
Chancro & $\begin{array}{c}\text { Caverna } \\
\text { prim. }\end{array}$ & $\begin{array}{c}\text { Carerna } \\
\text { noprim. }\end{array}$ & $\begin{array}{c}\text { Ganglio } \\
\text { caseoso }\end{array}$ & Totales \\
\hline
\end{tabular}

Bronquio D.

br. princlp.

5

3

br. secundar.

\section{Bronquio I.}

br. princip.

br. secundar. 1

Esófago

Tráquea

Pericardio

Pleura

Mediastino

Totales

1

.

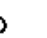

1
1

2 el aspecto macroscópico de la lesión intestinal era típica de úlceras "de salida" (por vía linfática retrógrada) en $18 \mathrm{ca}$ sos, por lo cual creemos que la cifra de $87 \%$ debe ser rebajada al $33.33 \%$. La causa de muerte más frecuente en los 43 casos a que nos estamos refiriendo, fué la tuberculosis miliar generalizada en 31 casos $(72.09 \%)$, sola (15 veces) o acompañada de meningitis tuberculosa (13 veces) o de tuberculosis acinosa exudativa palmonar bilateral ( 3 veces). Los 12 casos restantes fallecieron por tuberculosis acinosa pulmonar bilateral ( $5 \mathrm{ca}-$ sos), tuberculosis crónica cavernosa pulmonar ( 3 veces), meningitis tuberculosa sola (1 vez), tuberculomas encefálicos múltiples (1 vez) y bronconeumonía cararral (2 veces).

En el resto de las perforaciones (esófago, pericardio, pleura y mediastino, 7 casos) la causa de muerte nada tuvo que ver con el accidente que tratamos, sino con la enfermedad pulmonar tuberculosa.

Como puede observarse, en la perforación bronquial existe un alto porcentaje $(76 \%)$, que" da lugar a la tuberculosis palmonar por aspiración del material bacilifero vaciado al lumen bronquial, pero la causa de muerte en estos
17

1

12

\begin{tabular}{rrr}
12 & 13 & 26 \\
& 1 & 2 \\
2 & 4 & 8 \\
1 & 1 & 2 \\
1 & 1 & 2 \\
& 1 & 2 \\
1 & 1 & 2 \\
\hline 35 & -50 &
\end{tabular}

mismos casos, en general no guarda relación con la tuberculosis broncógena, sigo con una diseminación hematógena (tuberculosis miliar generalizada y meningitis tuberculosa), enfermedades estas últimas que pueden tener su origen en el sitio mismo de la lesión destructiva, pero también puede estar en el resto de la adenopatía caseosa que acompaña al causante de la perforación y que, según hemos visto, es de considerable extensión. Por otra parte, debemos tomar en cuenta que en 10 casos de perforaciones bronquiales $(23.26 \%$ ) no encontramos propagación canalicular, existiendo los medios materiales externos para ello, lo que puede estar en relación con condiciones internas del sujeto, que tengan que ver con sus medios humorales y tisurales de defensa contra los bacilos de Kocb. Es probable que todos estos factores (externos o internos), que el clínico no siempre puede apreciar debidamente, jueguen un papel importante en la evolución de estos casos y a ello se deba la discordancia con que los autores se refieren al pronóstico de la perforación bronquial, totalmente fatal para algunos (Huebschmann ${ }^{20}$, Beitzke ${ }^{21}$, etc.) y de curso menos grave y hasta favorable para otros (Weigert ${ }^{22}$, Ghon ${ }^{17}$, etc.). 
V

Tuberculosis miliar generalizada.

Esta enfermedad, can conocida morfológica y clínicamente, pero de patogenia aun muy discutida, adquiere importancia relevante en los primeros años de la vida. De reconocido origen hematógeno, puede presentarse en niños con complejo primario activo, en evolución ("generalización precoz"), de Huebschmann ${ }^{2 t}$, 0 aparecer más tarde, a partir de focos de tuberculosis orgánica que puede observarse su mayor frecuencia en los 3 primeros años de la vida, en los cuales fluctía entre el 72 y $74 \%$. Igualos resultados anota Slayton y Asset$s^{26}{ }^{26}$ en 135 casos de miliar generalizada, corroborados también por Wollstein y Bartle ${ }^{31}$, para quienes "mientras más joven es el paciente, más generalizada es la infección tuberculosa". Desde los 3 años, las cifras descienden gradualmente hasta los 7 años, edad esta última donde existe un porcentaje de $38 \%$ más o menos. A los 8 años la frecuencia de esta enfermedad aumenta al $62 \%$, para des-

Cuadro $N^{9} 18$

Porcentaje de la tuberculosis miliar generalizada, según la edad y sexo.

\begin{tabular}{|c|c|c|c|c|c|}
\hline \multirow[t]{2}{*}{ Edades } & \multirow{2}{*}{$\begin{array}{l}\mathrm{N}^{*} \text { autop. } \\
\text { con the. }\end{array}$} & \multirow{2}{*}{$\begin{array}{l}\text { Casos } \\
\text { posit. }\end{array}$} & \multirow{2}{*}{$\begin{array}{c}\text { Porcen- } \\
\text { taje }\end{array}$} & \multicolumn{2}{|c|}{ Porcentaje } \\
\hline & & & & Mascul. & Femen. \\
\hline $0-12 \mathrm{~ms}$. & 94 & 68 & 72.34 & 45.83 & 54.16 \\
\hline $12-24 \mathrm{~ms}$. & 99 & 73 & 73.73 & 57.53 & 42.46 \\
\hline 2- 3 a. & 62 & 46 & 74.19 & 52.17 & 47.82 \\
\hline 3- 4 a. & 37 & 25 & 67.515 & 52 & 48 \\
\hline 4- 5 a. & 31 & 20 & 64.51 & 45 & 55 \\
\hline 5- 6 a. & 21 & 10 & 47,61 & $2 p$ & 80 \\
\hline 6.7 a. & 29 & 11 & 37.03 & 54.54 & 45.45 \\
\hline 7- 8 a. & 16 & 10 & 62.50 & 60 & 40 \\
\hline 8- 9 a. & 17 & 10 & 158.82 & 20 & 80 \\
\hline 9-10 a. & 17 & 10 & 58.82 & 88.88 & 11.11 \\
\hline $10-11$ a. & 31 & 13 & 41.03 & 69.23 & 30.76 \\
\hline 11-12 a. & 16 & 8 & 50 & 66.66 & 33.33 \\
\hline $12-14$ a. & 6 & 4 & e6.66 & 100 & - \\
\hline 14-16 a. & 2 & 0 & - & 一 & - \\
\hline Totales & 478 & 308 & & & \\
\hline
\end{tabular}

irrumpen al sistema circulatorio ("generalización tardia" de Huebschmann). En el primer caso es el propio complejo primario la fuente de infección de la sangre $y$ en el segundo se trata de focos de tuberculosis orgánica ("tubérculo de la íntima" de Weigert ${ }^{22}$, focos caseosos del epidídimo, cápsulas suprarrenales, etc.), que se vacian secundariamente al torrento sanguíneo,

La frecuencia de la tuberculosis miliar generalizada en nuestro materia? (cuadro $\mathrm{N}^{\circ} 18$ ) alcanza un promedio de $60.44 \%$. Según la edad de los casos, cender nuevamente hasta los 11 años, donde alcanza el $42 \%$. Finalmente, a los 12 años existe una nueva alza $(50 \%)$, alcanzando a los 14 años la cifra de $66.5 \%$. En el cuadro $N^{\circ} 19$, ia curva de frecuencia de la lesión que comentamos, se manifiesta muy claramente en el sentido de un franco descenso a los 7 y 11 años, existiendo antes y después de las edades indicadas, ascensos muy evidentes.

Respecto del sexo, éste es variable, según las diferentes edades, aunque en general predomina el masculino. El dato 
global indica mayor existencia del masculino (164 y 148 casos, respectivamente), dando un porcentaje de 52.46 a favor de aquel sexo.

Cuodro $N 0.19$

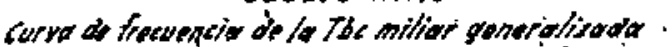

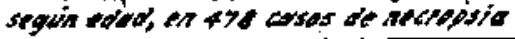

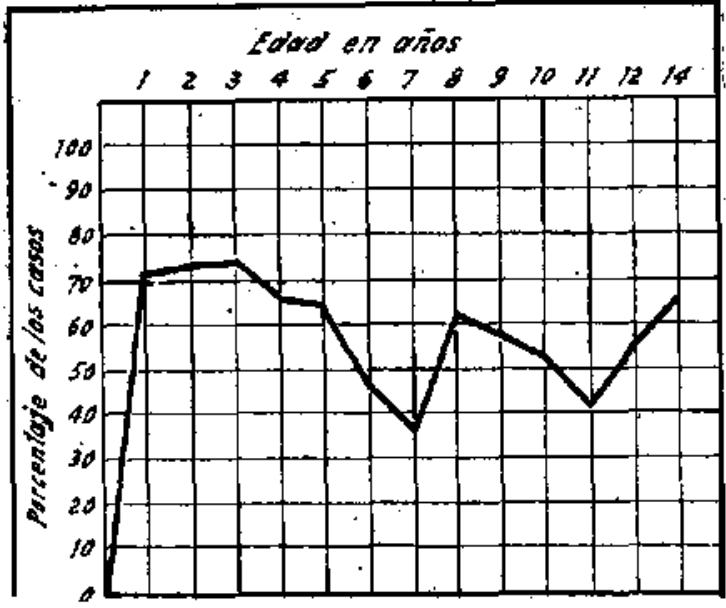

Como decíamos al empezar este párrafo, los autores consideran como factor etiológico de la miliar genéralizada, el complejo primario para la "generalización precoz" de Huebschmann y los focos de tuberculosis orgánica para la forma posterior al complejo primario. En \& cuadro $\mathrm{N}^{\circ} 20$ resumimos los diferentes factores etiológicos, cuyos porcentajes

Cuadro $\mathrm{N}^{\mathrm{P}} 20$

Factores etiológicos en Ja tuberculosis miliar generalizada. Porcentaje de tales factores en relación con los existentes en 478 cadáveres de tuberenlosos.

$N^{\circ}$ casos Su coexisen todo el tencla con material mil. gener.

Chancros caseifica.

$$
\text { dos }
$$

Gavernas en general

Adenopatía caseosa

Adenopatia genera. lizada

194

57

141

22

277

184

Perforaclones en general

Tbc. orgánicas

Propagacion intrabrongutal
$\%$

\section{.}

son decididamente elocuentes respecto de la importancia que éstos pueden tener en la génesis de la enfermedad que comentamos. Casi todos estos factores los obcervamos en cifras elevadas, superiores a! $70 \%$, lo que, a nuestro entender, revela una efectiva participación en aquel proceso. El mecanismo intimo de la diseminación hematógena, irrupción de caseum ganglionar al sistema circulatorio, no siempre es posible verificarlo en la disección anatómica, aunque con alguna frecuencia se le ha observado en el ángulo venoso formado por la yugular interna y el tronco braquiocefálico (Aschoff $\mathrm{f}^{2}$ y otros). Por lo demás, nada se opone a que ganglios caseificados viertan su producto bacilifero en un vaso sanguíneo de cualquicr territorio del organismo, así como también el chancro pulmonar, una Ferforación torácica o un foco caseoso de ura tuberculosis orgánica (riñón, epidídimo, hueso, etc.) lo haga a un vaso vecino, directamente o por intermedio de los linfáticos eferentes, con idénticas consecuencjas.

En general, los autores consideran que son los ganglios los órganos que con mayor frecuencia dan lugar a la miliar generalizada y entre éstos hacen figurar, en primer lagar, a los del mediastino (Peña y Feldman ${ }^{23}$. La existencia en nuestro material de fuentes múltiples para la producción de esta enfermedad, hace dificil el otorgar rango etiológico preferente a determinadas causas y sólo podemos concluir que todas ellas constituyen causas potenciales, que en un momento dado pueden ser capaces, en forma simultánea o intermitente, de dar lugar a una diseminación hematógena.

\section{VI}

\section{Lepto-meningitis tubercalosa.}

Esta enfermedad la bemos encontrado en 200 casos de 478 niños fallecidos con lesiones tuberculosas, o sea, en el $41.84 \%$. Su frecuencia, en relación con la edad (cuadros 21 y 22), se observa que asciende desde los primeros meses (26\%) hasta el $4^{\circ}$ año de vida $(54 \%)$. 
Cuadro $\mathrm{N}^{\circ} 21$

Lepto-ineningitis tuberculosa. Edad, sexo y frecuencia respectiva.

Edades $N^{9}$ Casos Porcen- Porcentaje
necrop. posit. taje Masc. Fem.

\begin{tabular}{|c|c|c|c|c|c|}
\hline D. $6 \mathrm{~ms}$. & 42 & 11 & 26.19 & 36.36 & 63.63 \\
\hline 6-12 ms. & 52 & 19 & 36.53 & 47.36 & 52,63 \\
\hline $12-18 \mathrm{~ms}$ & 53 & 18 & 33.96 & 45 & 55 \\
\hline 18-24 ms. & 46 & 18 & 39.13 & 47.21 & 52.63 \\
\hline $2-3 a$. & 62 & 33 & 53.22 & 53.12 & 46.87 \\
\hline $3-4 a$. & 37 & 20 & 54.05 & 42.10 & $57: 39$ \\
\hline 4- 5 a. & 31 & 14 & 45.16 & 57.14 & 42.35 \\
\hline $5-6 a$. & 21 & 11 & 52.38 & 18.18 & 81.81 \\
\hline 6- $7 \mathrm{a}$. & 29 & 11 & 37.93 & 50 & 50 \\
\hline $7-8 a$. & 16 & 7 & 43.75 & 71.42 & 28.57 \\
\hline 8- 9 a. & 17 & 9 & 52.94 & 50 & 50 \\
\hline $8-10 \mathrm{a}$. & 17 & 11 & 64.70 & 72.72 & 27.27 \\
\hline 10-11 a. & 31 & 9 & 29.03 & 75 & 25 \\
\hline 11-12 a. & 16 & 7 & 43.75 & 100 & - \\
\hline 12-13 a. & 4 & 2 & 50 & 100 & - \\
\hline 13-16 a. & 4 & 0 & & & \\
\hline Totales & 478 & 2000 & $4 \%)$ & & \\
\hline
\end{tabular}

En este mismo lapso (o a 4 años) se encuentra también el $60 \%$ de los casos de meningitis (119 entre 200). Proporciones análogas citan en estadisticas anátomo-patológicas Simón y Redeker ${ }^{3}$, Slayton y Asserson ${ }^{28}$, Neal ${ }^{27}$, Koch ${ }^{28}$, etc. La mayor frecuencia en este período de la vida la explica Engel ${ }^{3}$ por las particula-

Cuaciro No.22

iurver de frecuencio de la leptomeningifis The seguin edord, en 478 casas de necropsia

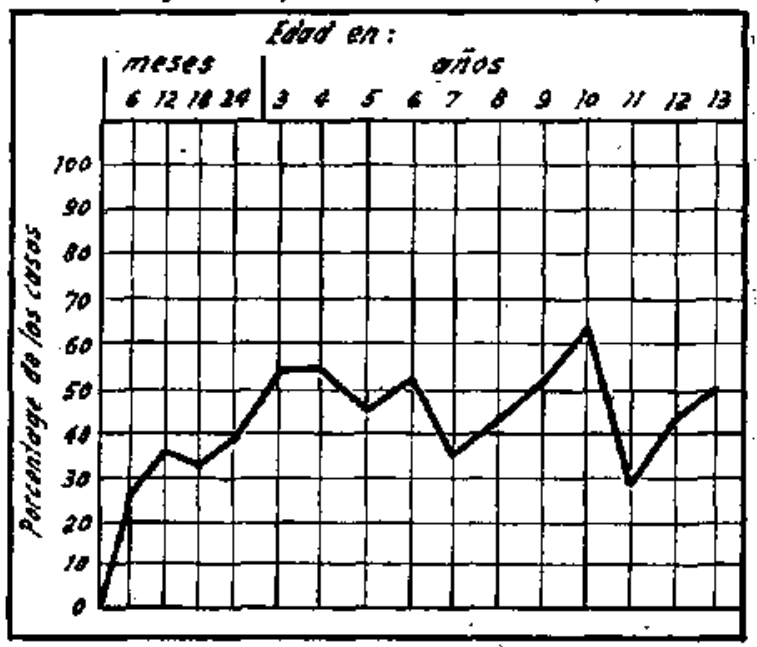

ridades del aparato linfático en tal edad, que favorece la extensión de la tuberculosis ganglionar (escrofulosis), la cual, a su vez, prepara el terreno para ia invasión tuberculosa vascular. Sobre los 4 años, los porcentajes son, en general, menores, hasta llegar a los 10 años, en que mievamente se produce una alza que al. canza al $65 \%$, después de los crales baja bruscamente al $29 \%$. Algunos autores, como Bonaba20, por ejemplo, han observado también igual descenso en la frecuencia de la meningitis a los 11 años, sin que se encuentre una explicación satisfactoria para este hecho.

Respecto del sexo, podemos afirmar que la meningitis tuberculosa afecta, con jigero predominio, al masculino (104 (52\%) y $96(48 \%)$, respectivamente) en el total de los casos con meningitis. Slayton y Asserson ${ }^{2 a}$, en 135 casos, encuentran proporciones semejantes: $54 \%$ para el masculino y $46 \%$ para el femenino. Sin embargo, en los dos primeros años de la vida, es evidente en nuestro material el mayor porcentaje de esta enfermedad en el sexo femenino, el cual fluctúa entre el 64 y el $53 \%$. Por al contrario, Schelling ${ }^{30}$ ha observado mayor número de casos masculinos en los primeros 4 años y sobre esta edad pre dominio del sexo femenino. En nuestra casuistica no encontramos sobre los 2 años una clara predominancia de un sexo sobre otro, según puede comprobarse en el cuadro $N^{\circ} 21$, a excepción del $5^{\circ}$ año de vida en que el sexo femenino alcanza la cifra de $80 \%$.

Existe consenso unánime que la mayoria de las meningitis tuberculosas se deben a diseminaciones hematógenas, por lo cual su fuente de origen es la misma que la de la tuberculosis miliar generalizada. Por esta razón, aquella enfermedad coexiste con la miliar generalizada en un porcentaje alto, según vetemos más adelante. Pero también puede producirse la infección tuberculosa de las meninges por propagación por continuidad, por ejemplo: tuberculoma encefálico o meníngeo, otitis $1 / 2$ tuberculosa, Osteitis de los huesos del cráneo, etc. Sin em- 
bargo, el factor que con mayor frecuencia la produce, de acuerdo con la opinión de varios autores, Engel ${ }^{5}$, Wangenheim ${ }^{22}$, Grcengard $^{33}$, etc, es la adenopatía tuberculosa caseosa proveniente del complejo primario. A esto se debería la preponderancia de esta enfermedad an años juveniles (Engel ${ }^{3}$ ). Efectivamente, este último autor, en su material necrópsico ba encontrado corrientemente la moningitis tuberculosa con complejo primario fresco $y$ en cantidad insignificante aquéllos involucionados o curados. A este respecto, nosotros bemos obtenido resultados más o menos análogos (cuadro $N^{\circ} 23$ ), pues el chancro caseoso esta-

Cuadro N 23

Estado físico del complejo primario en 200 casos de meningitis tuberoulosa.

\begin{tabular}{|c|c|c|}
\hline & $\mathbb{N}^{\circ}$ total & Porcentajes \\
\hline Chancros caseosos & 98 & 80.32 \\
\hline $\begin{array}{l}\text { Chancros eretáceos } \\
\text { calcificados }\end{array}$ & 24 & 19.67 \\
\hline No se encontró & 78 & \\
\hline $\begin{array}{l}\text { Adenopatía caseosa } \\
\text { Adenopatia cretácea }\end{array}$ & 170 & 89.94 \\
\hline $\begin{array}{l}\text { calclficada } \\
\text { No se encontró }\end{array}$ & $\begin{array}{l}19 \\
11\end{array}$ & 10.05 \\
\hline
\end{tabular}

ba presente en el $80 \%$ y la adenopatía caseosa en el $90 \%$ de los casos con meningitis tuberculosa. Añade Engel que la tuberculosis pulmonat manifiesta (propagaciones bronquiales neumónicas o Eronconeumónicas, cavernas crónicas, etc.) la considera en escasa relación con la génesis de la meningitis taEerculosa, concluyendo que la meningitis tuberculosa "sería la manera típica con que llegan a la muerto las tuberculosís ocultas". En nuestro material, también las tuberculosis pulmonares manifiestas tienen una mínima incidencia, pues sólo alcanzan al $6.5 \%$ en el total de casos con meningitis tuberculosa. En resumen, según nuestros hallazgos, podemos atribuir a los elementos del complejo primario la génesis de la mayoría de las meningitis, 170 de nuestros 200 casos, o sea, el $85 \%$. En la mitad de estos 170 casos (85 casos) la parte ganglionar tevelaba una reactivación franca del proceso tuberculoso, pues se encontraba extendida a zonas vecinas (región cervical y abdominal). De acuerdo con el cuadro 23, ya citado, encontramos también cierto porcentaje, aunque pequeño, en que el complejo primario estaba cretificado o calcificado iol $20 \%$ de los chancros y

\section{Cuadro N: 24}

\section{Relación global de las diseminaciones hema: tógenas y meningitis tuberculosas según edad.}

\begin{tabular}{|c|c|c|c|c|}
\hline Edades & $\begin{array}{c}\mathrm{N} \\
\text { total }\end{array}$ & $\begin{array}{l}\text { Mil. gen. } \\
\text { c. mening. }\end{array}$ & $\begin{array}{l}\text { Mil.gen. } \\
\text { s. mening. }\end{array}$ & $\begin{array}{c}\text { Mening } \\
\text { sola }\end{array}$ \\
\hline 0-12 ms. & 68 & 30 & 38 & 0 \\
\hline $12-24 \mathrm{~ms}$. & 76 & 33 & 40 & 3 \\
\hline 2- 3 a. & 51 & 28 & 18 & 5 \\
\hline $3-4 a$. & 26 & 19 & 6 & 1 \\
\hline 4- 5 a. & 22 & 12 & 8 & 2 \\
\hline 5- $6 a$. & 15 & 6 & 4 & 5 \\
\hline 6. $7 \mathrm{a}$. & 16 & $\theta$ & 5 & 5 \\
\hline $7-8 a$ & 10 & 7 & 3 & - \\
\hline 8-9a. & 13 & 6 & 4 & 3 \\
\hline $9-10$ a. & 13 & 8 & 2 & 3 \\
\hline 10-11 a. & 13 & 9 & 4 & - \\
\hline 11-12 a. & 9 & 6 & $\mathbf{2}$ & 1 \\
\hline 1'2-13a. & 4 & 2 & 2 & - \\
\hline Totales & 336 & 172 & 136 & 28 \\
\hline
\end{tabular}

el $10 \%$ de las adenopatías), lo que indica en general, salvo las excepciones que luego añadiremos, que otras lesiones tuberculosas independientes del complejo primario, participaron en el desarrollo de la enfermedad que comentamos. Sin embargo, debemos hacer presente que entre estos complejos involucionados, 4 habían sido tratados intensamente por estreptomicina, a lo cual podemos atribuir la cretificación o calcificación. Estos 4 casos eignifican $3.37 \%$ y $2.12 \%$ correspondientes a los chanctas y ganglios, respe:tivamente, porcentajes que sin estreptomicinoterapia se habrían agregado a los elementos caseificados, con la reducción consiguiente de los chancros y ganglios cretificados o calcificados. En todo caso, 
persiste cierta proporción de casos con meningitis tuberculosa, cuyo complejo primario está involucionado, en oposición a lo manifestado por Enge ${ }^{5}$, que en su estadística apenas si lo encuentra.

Como decíamos anteriormente, la meningitis tuberculosa coexiste con la miliar generalizada en porcentajes altos, variables según los autores: para Simón y Redeker $^{3}$ es del $93 \%$, para Huebschman ${ }^{2}$ y Terplan' ${ }^{34}$ es del $75 \%$, para Bartlet y Wolstein ${ }^{35}$ del $40 \%$, etc. En nuestra casuística (cuadros Nos. 24 y 25) la proporción global alcanza al $56 \%$ más o menos. Los porcentajes de esta coexistencia, según las diferentes edades (cuadro $\mathrm{N}^{\circ} 25$ ), aparecen gradualmente as-

\section{Cuadro $\mathbf{2 5}$}

Coexistencia de Ia meningitis tuberculosa en el total de tuberculosis miliar generalizada (308 casos).

\begin{tabular}{|c|c|c|c|}
\hline Edades & $\begin{array}{l}\mathbf{N}^{\circ} \text { total de } \\
\text { mil. gen. }\end{array}$ & $\begin{array}{l}\text { Mil. gen. } \\
\text { c. menlng. }\end{array}$ & $\begin{array}{l}\text { Mil, gen. } \\
\text { s. mening. }\end{array}$ \\
\hline $0-12 \mathrm{~ms}$. & 68 & $44.11 \%$ & $55.88 \%$ \\
\hline $12-24 \mathrm{~ms}$. & 73 & $45.20 \%$ & $54.79 \%$ \\
\hline 2- 3 a. & 46 & $60.86 \%$ & $39.13 \%$ \\
\hline $3-4 a$ & 25 & $76 \%$ & $24 \%$ \\
\hline 4- $5 \mathrm{a}$. & 20 & $60 \%$ & 40 \\
\hline $5-6 \mathrm{a}$. & 10 & $60 \%$ & $40 \%$ \\
\hline $6-7 a$. & 11 & $5454 \%$ & $45.45 \%$ \\
\hline $7-8 a$ & 10 & $70 \%$ & 30 \\
\hline $8-9$ a. & 10 & $60 \%$ & 40 \\
\hline $9.10 \mathrm{a}$. & 10 & $80 \%$ & $20 \%$ \\
\hline 10-11 a. & 13 & $60.23 \%$ & $30.76 \%$ \\
\hline $11-12$ a. & 8 & $75 \%$ & $25 \%$ \\
\hline $12-13$ a. & 4 & 50 & 50 \\
\hline Total & 308 & & \\
\hline
\end{tabular}

cendentes desde 0 mes hasta los 4 años, edad esta última en que alcanza el $76 \%$. Aunque en las edades posteriores las cifras decrecen, encontramos a los 10 años an evidente ascenso al $80 \%$. Si comparamos nuestros resultados con los de Huebschmann (cuadros Nos. 26 y 27 ), podemos observar, especialmente en el cuadro $N^{2} 27$, que los porcentajes nuestros están muy por debajo de los de Huebschmann, más o menos en la mitad
Cuadro NP 26

Cuadro comparativo entre los resultados de Huebschmann y los nuestros respecto de la diseminación hematógena y meningitis tuberculosa, según edad.

\begin{tabular}{llll}
\hline Edades & $\begin{array}{l}\text { No total Mil. } \\
\text { de casos }\end{array}$ & Men. & Merning. \\
& c. men. & sola \\
&
\end{tabular}

0-12 meses

$\begin{array}{lrrrr}\text { Huebschmann } & 25 & 18 & 4 & 2 \\ \text { Nosotros } & 68 & 30 & 38 & 0\end{array}$

1- 5 años

$\begin{array}{lllll}\text { Huebschmann } & 89 & 67 & 6 & 16\end{array}$

$\begin{array}{lllll}\text { Nosotros } & 175 & 92 & 72 & 11\end{array}$

6-10 años

Huebschmann $26 \quad 23 \quad 003$

$\begin{array}{lllll}\text { Nosotros } & 67 & 33 & 18 & 16\end{array}$

Cuadro $\mathrm{N}^{\circ} 27$

Cuadro comparativo entre los resultados de Huebschmann y los nuestros, respecto del porcentaje de coexistencia de la meningitis tuberculosa con la tuberculosis miliar generalìvada, según edad.

\begin{tabular}{rrr}
\hline $\begin{array}{l}N^{\circ} \text { total } \\
\text { mill. gen. }\end{array}$ & c. mening. & Mil. gen. \\
\hline
\end{tabular}

0-12 meses

Huebschmann $23 \quad 82.60 \% \quad 17.39 \%$ Nosotras $\quad 68 \quad 44.11 \% \quad 55.88 \%$

1. 5 años

Huebschmann $73 \quad 91.78 \% \quad 8.21 \%$ Nosotros $\quad 164 \quad 56.09 \% \quad 43.90 \%$

6-10 afios

$\begin{array}{lccc}\text { Huebschmann } & 23 & 100 \% & \\ \text { Nosotros } & 51 & 64.70 \% & 35.29 \%\end{array}$

ya que en el material de este autor la coexistencia dé las enfermedades que comentamos oscilan del 82 al $100 \%$, cifra esta última que alcanza en el lapso de 6 a 10 años. Debemos agregar que del grupo de los fallecidos con miliar generalizada (308 en total), sólo 5 fueron tratados con dosis importantes de 
estreptomicina, pese a lo cual no se encontraron modificaciones morfológicas en lo que a miliar se refiere, aunque pudo tener influencia en la prevención o falta de desarrollo de la meningitis tuberculosa, pero que por su traducción porcentual $(1.62 \%)$ creemos puede desestimarse.

Ell el grupo de las meningitis tuberculosas (200 casos), encontramos 28 casos en que esta enfermedad no coexistía con miliar generalizada (cuadro $\mathrm{N}^{\circ} 24$ ), lo cual corresponde a un porcentaje global de 14. Analizadas segúm las odades en que esto ocurre (cuadro 28), podemos

\section{Cuadro $\mathrm{N}^{\circ} 28$}

Frecuencia de meningitis tuberculosa sola (sin miliar), en el total de meningitis tuberculosa (200 casos).

\begin{tabular}{lll} 
Edades & $\begin{array}{l}N^{\circ} \text { total } \\
\text { mening. }\end{array}$ & $\begin{array}{c}\text { Mening. } \\
\text { sola }\end{array}$ \\
\hline
\end{tabular}

\begin{tabular}{|c|c|c|c|}
\hline $0-12 \mathrm{~ms}$ & 30 & 0 & 0 \\
\hline $12-24 \mathrm{~ms}$. & 36 & 3 & .8 .33 \\
\hline 2- 3 a. & 33 & 5 & 15.15 \\
\hline $3-4 a$. & 20 & 1 & 5 \\
\hline $4-5 \mathrm{a}$. & 14 & 2 & 14.28 \\
\hline 5- 6a. & 11 & 5 & 45.45 \\
\hline 6- $7 \mathrm{a}$. & 11 & 5 & 45.45 \\
\hline $7-8 \mathrm{a}$. & 7 & 0 & 0 \\
\hline 8- 9 a. & 9 & 3 & 33.33 \\
\hline 9-10 a. & 11 & 3 & 27.27 \\
\hline 10-11 a. & 9 & 0 & 0 \\
\hline $11.12 \mathrm{a}$. & 7 & 1 & 14.28 \\
\hline 12-13 a. & 2 & $\mathbf{0}$ & \\
\hline Total & 200 & 28 & \\
\hline
\end{tabular}

observar que su mayoría está sobre los 5 años de edad, lo cual podría relacionarse con el lapso en que la miliar generalizada disminuye realmente de frecuencia (cuadro $\mathrm{N}^{9} 18$ ). Si comparamos este hallazgo con los datos respectivos de Huebschmann (cuadro $\mathrm{N}^{\circ} 29$ ), encontramos que este autor tiene un porcentaje global de meningitis tuberculosa sola, igual a 16.15 variable en las diferentes edades, pero repartidas en cantidades un tanto aproximadas en los diversos lapsos. En cambio, los casos nuestros están ausentes en el primer año y tienen sa máximo en la etapa de 6 a 10 años (35\%). Debemos también aquí hacer presente que de nuestros 28 casos, 11 fueron tratados intensamente con estreptomicina, a lo cual podríamos atribu ir su no coexistencia con miliar generalizada. Sin embargo, sólo en 5 de estos 11 casos, se encontró macro y microscópicamente escasos tubérculos aislados en uno o dos órganos (hígado, bazo, pulmón, pleura, etc.), muchos de ellos fibrosados - calcificados, como expresión de una miliar curada. Nos asisten dudas que en los otros 6 casos, si hubo tuberculosis miliar, haya podido curar tan absolutamente, sin dejar siquiera algunos gránulos fibrosados como en los casos precedentes. Según esto, podríamos rebajar en el grupo de meningitis tuberculosas solas, los 11 casos tratados con estreptomicina 0 solamente los 5 que por las secuelas encontradas hacen suponer la existencia de miliar generalizada, con lo cual la frecuencia general de esta forma particular de meningitis, descendería del $14 \%$ al $8.5 \%$ o al $12 \%$, respectivamente. Los resultados de estas modificaciones en el cuadro $\mathrm{N}^{\circ} 29$, se copian entre paréntesis.

\section{Cuadro $N^{\circ} 29$}

Cuadro comparativo de los resultados de Huebschmann con los nuestros, respecto de la frecuencia de la meningitis tuberculosa sola (en el material de meninsitis tuberculosa), según edad.

Edades $\begin{gathered}\text { Notal }^{\circ} \\ \text { mening. }\end{gathered} \quad \begin{gathered}\text { Mening. } \\ \text { sola }\end{gathered}$

0-12 meses

Huebschmann $21 \quad 9.52 \%$

Nosotros $30 \quad 0 \%$

1- 5 años

Huebschmann $83 \quad 19.27 \%$

Nosotros $\quad 103 \quad 10.67 \%(8.73 \%)$

6-10 años

Huebschmann $26 \quad 11.69 \%$

Nosotros $\quad 49 \quad 34.69 \%(14.2 B \%-28.53 \%)$ 
El becho que la miliar generalizada no afecte en algunas ocasiones a las menizges y especialmente que se desarrolle meningitis tuberculosa sin tuberculosis miliar generalizada, hizo idear a $\mathrm{Rich}^{88}$ una teoria según la cual la meningitis tuberculosa es, en todos los casos, una tuberculosis del espacio sub-aracnoídeo, que contiene líquido céfalo-raquídeo. Este espacio podría infectarse directamente descle la sangre, lo cual este autor considera raro. dando lugar a una meningitis tuberculosa como síntoma parcial de la miliar hematógena, o lo que cree más frecuente, dicho espacio se infecta desde el cerebro mismo por un foco tuberculoso antiguo (tuberculoma), producido por igual mecanismo que la llamada tuberculosis orgánica (vía hematógena). El tuberculoma puede encontrarse ubicado en el cerebro mismo o médula espinal, en los plexos coroídeos, en las envolturas meníngeas cerebro-medulares o en las cavidades óseas vecinas. En apoyo de sll teoria, $\mathrm{Rich}^{38}$, mediante cuidadosa laminación del cerebro, ha encontrado tuberculomas cerebrales en el $94 \%$ de sus meningitis. Otros investigadores dan resultados menores, probablemente porque la técnica de la búsqueda del tuberculoma necesita suma prolijidad, ya que es menester laminar todo el sistema nervioso central y observar lesiones que suelen ser muy pequeñas. Así, por ejemplo, Mac Gregor y Green $^{\text {a7 }}$ han encontrado tuberculomas en el $74 \%$ de las meningitis, Ragins ${ }^{\Im i}$ en el $41 \%$, Schuermann ${ }^{37}$ en el $36.8 \%$, Redman ${ }^{\text {a7 }}$ en el $30 \%$, etc. En nuestro país, Saldías ${ }^{88}$ en 28 autopsias de meningitis tuberculosa encontró 8 tuberculomas, o sea, en el $28.57 \%$, utilizando la técnica corriente y Schwarz ${ }^{89}$ en el $41.20 \%$ entre 24 autopsias de meningitis tuberculosa. En nuestro material de 200 autopsias de meningitis tuberculosa, hemos encontrado, sin la técnica seguida por Rich, 29 casos con tuberculoma, lo que significa una frecuencia del $14.5 \%$, hallazgo que por la técrica usada creemos que no corresponde a la realidad. En el cuadro $N^{\circ} 30$, que resume los tubercu-

$$
\text { Cuadro N" } 30
$$

Frecuencla de tubercalomas en la meningitis tuberculosa, según edad.

\begin{tabular}{c}
\hline Edades Casos \\
$\begin{array}{c}\text { c. men. } \\
\text { tbc. }\end{array}$
\end{tabular}

$\begin{array}{rrrrr}0-6 \mathrm{~m} . & 11 & 1 & - & 9.09 \\ 6-12 \mathrm{~m} . & 18 & 2 & - & 10.52 \\ 12-18 \mathrm{~m} . & 18 & 2 & 1 & 16.66 \\ 18-24 \mathrm{~m} . & 18 & 2 & 1 & 18.16 \\ 2-3 \mathrm{a} . & 33 & 7 & 1 & 24.24 \\ 3-4 \mathrm{a} . & 20 & 4 & - & 20 \\ 4-5 \mathrm{a} . & 14 & - & - & 0 \\ 5-8 \mathrm{a} . & 11 & - & 1 & 9.09 \\ 6-7 \mathrm{a} . & 11 & 4 & - & 36.36 \\ 7-8 \mathrm{a} . & 7 & 1 & - & 14.28 \\ 8-9 \mathrm{a} . & 9 & - & - & 0 \\ 9-10 \mathrm{a} . & 11 & - & 1 & 9.09 \\ 10-11 \mathrm{a} . & 0 & - & - & 0 \\ 11-12 \mathrm{a} . & 7 & 1 & - & 14.28 \\ 12-13 \mathrm{a} . & 2 & - & - & 0 \\ & - & - & - & \end{array}$

lomas encontrados en las meningitis tuberculosas, podemos observar que su mayoría (2l casos) se encuentran en los primeros 4 años. De los 29 casos, 24 $(82.75 \%)$ corresponden al encéfalo y $5(17.24 \%)$ a las meninges. Finalmente, de las 28 meningitis sin miliar generalizada, sólo $5(17.85 \%)$ revelaron ta existencia de tuberculomas. Si consideramos que el total de meningitis tuberculosas es de 200 casos, esta enfermedad no coexistía con tuberculomas en 171 casos, o sea, en el $84.50 \%$, cifra que, como dijimos anteriormente, no la consideramos real, ya que en la búsqueda de las tuberculomas no se siguió la técnica utilizada por Rich. 